\title{
Electron Spin Beats in InGaAs/GaAs Quantum Dots
}

\author{
(C) V.K. Kalevich, M.N. Tkachuk, P. Le Jeune*, X. Marie*, T. Amand*
}

A.F. Ioffe Physico-technical institute of Russian Academy of Sciences, 194021 St. Petersburg, Russia

* Laboratoire de Physique de la Matiere Condensée CNRS-UMR 5830 INSA, Complexe scientifique de Rangueil, 31077 Toulouse cedex, France

E-mail: kalevich@solid.ioffe.rssi.ru

\begin{abstract}
Time resolved picosecond spectroscopy is used for the first time to study optical orientation and spin dynamics of carriers in self-organized $\operatorname{In}(\mathrm{Ga}) \mathrm{As} / \mathrm{GaAs}$ quantum dots (QDs) arrays. Optical orientation of carriers created by 1.2 ps light pulses both in the GaAs matrix and wetting layer and captured by QDs is found to last a few hundreds of picosecond. The saturation of electron ground state at high excitation light intensity leads to electron polarization in excited states close to $100 \%$ and to its vanishing in ground state. Electron spin quantum beats in a transverse magnetic field are observed for the first time in semiconductor QDs. We thus determine the quasi-zero-dimensional electron $g$-factor in $\mathrm{In}_{0.5} \mathrm{Ga}_{0.5} \mathrm{As} / \mathrm{GaAs}$ QDs to be: $\left|g_{\perp}\right|=0.27 \pm 0.03$.
\end{abstract}

Semiconductor structures with quantum dots are currently the subject both of fundamental studies and of practical applications. In the last few years much attention was given to the study of the energy relaxation of carriers in those structures [1-7]. Taking into account the spin of carriers makes it possible to obtain additional information on the energy relaxation processes and the structure of the electron energy levels. In the present work, time resolved spectroscopy with picosecond resolution has been used for the first time to investigate spin dynamics in semiconductor quantum dots embedded in a semiconductor matrix. Spin polarization of carriers was created as a result of their optical orientation [8] by short ( $\sim 1 \mathrm{ps})$ pulses of circularly polarized light. It has been found that the spin polarization of electrons generated in the matrix or in the wetting layer, can survive their capture by the QDs and last a few hundreds of picoseconds. This allowed us to observe quantum beats of electron spin in a transverse magnetic field and thus to determine the magnitude of the transverse g-factor of quasizero-dimensional electrons in $\mathrm{In}_{0.5} \mathrm{Ga}_{0.5} \mathrm{As} / \mathrm{GaAs}$ quantum dots.

The structures studied were grown by solid-source molecular beam epitaxy in Riber-32P machine on semi-insulating GaAs (100) substrates. Active region was inserted into the middle of a $0.2 \mu \mathrm{m}$ thick undoped GaAs layer confined by $\operatorname{AlAs}(2 \mathrm{~nm}) / \operatorname{GaAs}(2 \mathrm{~nm})$ superlattices to prevent escape of non-equilibrium carriers to the sample surface and substrate. In the first structure, denoted as 1, the active region consists of 6 planes of QDs separated by $50 \AA$ thick GaAs spacers. Each QD plane was formed by deposition of 4 monolayers (MLs) of $\mathrm{In}_{0.5} \mathrm{Ga}_{0.5} \mathrm{As}$. In the structure 2 the active layer consists of 10 planes of $1.7 \mathrm{ML}$ InAs QDs separated by $200 \AA$ thick GaAs spacers. Sample 3 contains one plane of 2.7 ML InAs QDs. The growth temperature was set to be $485^{\circ} \mathrm{C}$ for In-containing layers and $600^{\circ} \mathrm{C}$ for the other parts of the structures [9].

A tunable Ti-sapphire laser producing 1.2-ps long light pulses with a repetition frequency of $82 \mathrm{MHz}$ was used to excite the investigated structures. The time resolution of the experimental setup, based on the parametric up-conversion technique, was limited by the laser pulse duration. The exciting circularly polarized light was obtained by passing linearly polarized lazer beam through a quarter-wave plate. The luminescence was registered along the growth axis $(\mathrm{Oz})$ in back-scattering geometry.

The results of polarization measurements in the different structures under study are qualitatively the same. Presented below are the results of experiments on sample 1. Fig. 1 shows normalized luminescence spectra obtained under excitation of carriers in the GaAs matrix. Spectrum 1 is recorded under continuous wave $(\mathrm{cw})$ excitation with a pump density of $1 \mathrm{~W} / \mathrm{cm}^{2}$. An increase of the cw excitation intensity by two orders of magnitude did not change the shape and position of the spectrum. This indicates that spectrum 1 results from the emission of ground-state electrons and holes. We associate the presence of two strongly overlapping lines in this spectrum with radiative recombination of two groups of dots having different mean

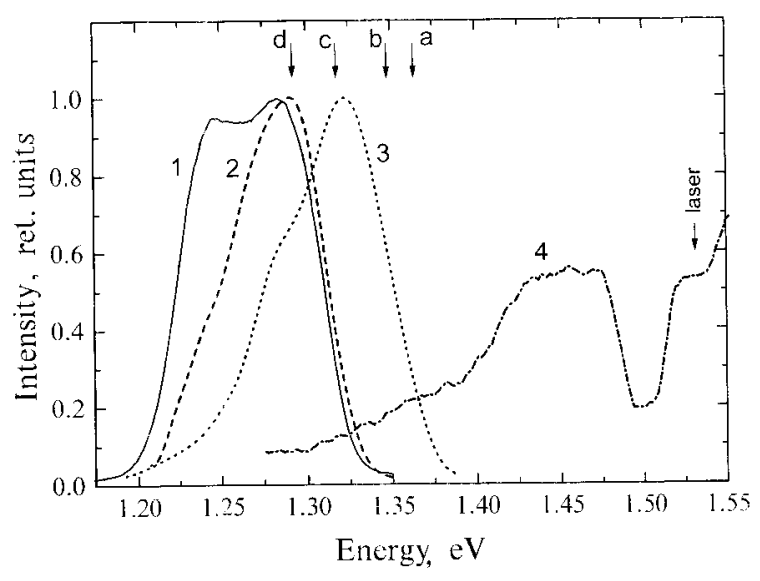

Figure 1. Photoluminescence spectra of $\operatorname{In}_{0.5} \mathrm{Ga}_{0.5} \mathrm{As} / \mathrm{GaAs}$ QDs (sample 1). $T=10 \mathrm{~K} .1-\mathrm{cw}$. excitation with $E_{\text {exc }}=1.610 \mathrm{eV}$ and power density $1 \mathrm{~W} / \mathrm{cm}^{2} ; 2$ and $3-$ short pulse pumping with power densities 0.1 and $1.6 \mathrm{MW} / \mathrm{cm}^{2}, E_{\text {exc }}=1.531 \mathrm{eV}$, time delay after excitation pulse equals $200 \mathrm{ps} ; 4-\mathrm{cw}$ photoluminescence excitation spectra at $E_{d e t}=1.248 \mathrm{eV}$. 


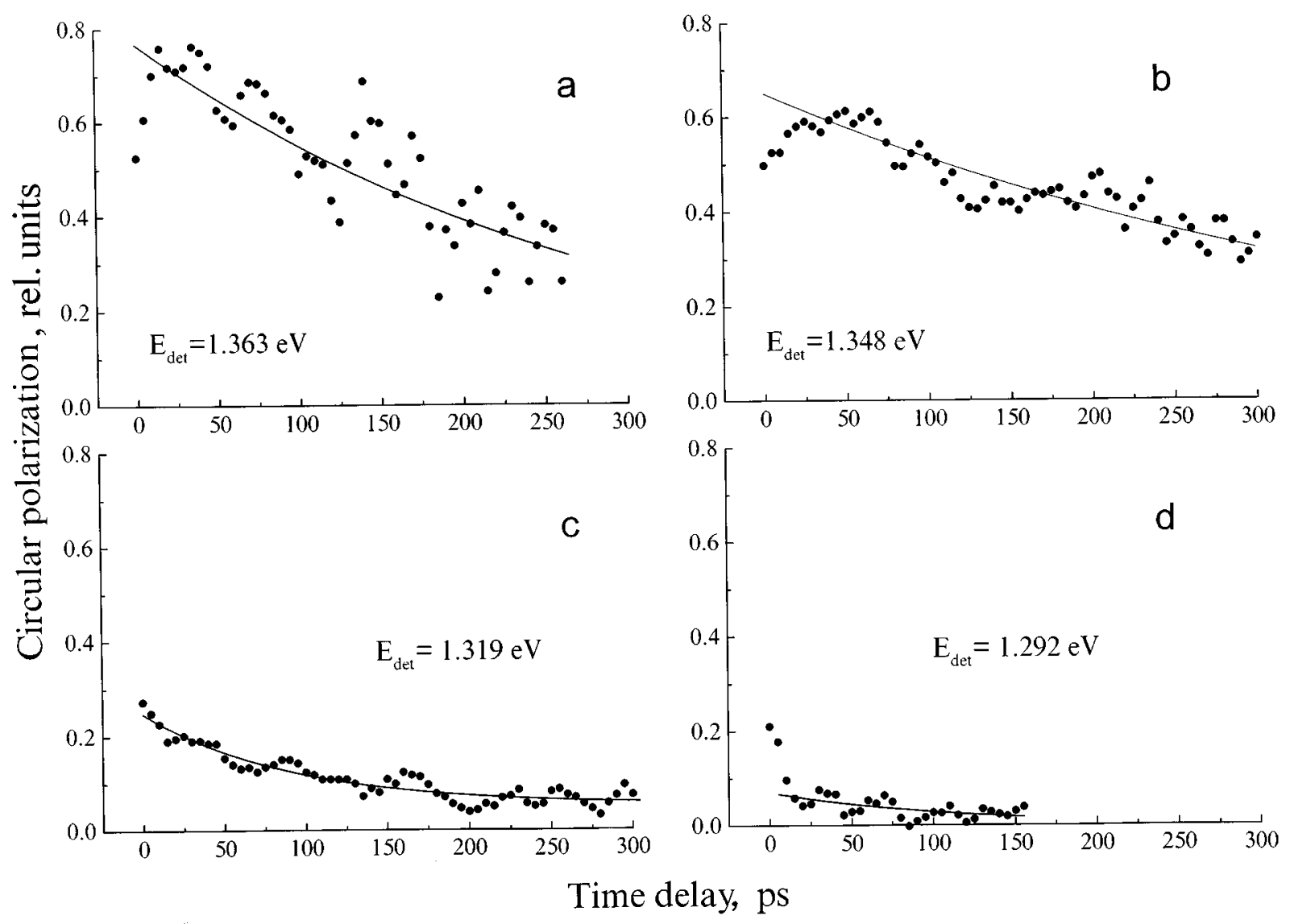

Figure 2. Luminescence circular polarization dependence on time delay at different detection energies $E_{\text {det }} . E_{\text {exc }}=1.531 \mathrm{eV}, T=10 \mathrm{~K}$. Solid lines show the exponential decay of the polarization degree with time constant $\tau_{S}$. $\tau_{S}(\mathrm{ps}): a-290, b-400, c-211, d-92$.

sizes. Spectra 2 and 3 are registered under pulse excitation with pump densities of 0.1 and $1.6 \mathrm{MW} / \mathrm{cm}^{2}$ respectively. As seen from the comparison with spectrum 1, an increase of the pulse excitation intensity results in a significant (up to $50 \mathrm{meV}$ ) blue shift of the luminescence line. Such transformation of the luminescence spectra of quantum dots at high excitation levels has been observed before $[4,6,7]$ and was due to the filling of the ground states of electrons and holes and to the appearance of an intense light emission from the excited states. Indeed, as an estimate shows, at the maximum pump density of $1.6 \mathrm{MW} / \mathrm{cm}^{2}$ that we used, the number of photoexcited electron-hole pairs exceeds by several times the number of quantum dots in the illuminated region; this leads to population of the excited states, since they cannot relax any more into the filled ground states. The excited states, in turn, give rise to an intense luminescence spectral component. Curve 4 in Fig. 1 represents the cw photoluminescence excitation spectrum recorded at a registration energy $E_{\text {det }}=1.245 \mathrm{eV}$ (the position of the lowenergy maximum in spectrum 1$)$. We, as well as the authors of $[4,7]$, assign the relatively broad band $(1.42-1.48 \mathrm{eV})$ near the edge of the fundamental absorption of GaAs to the absorption of the wetting layer.
In our experiments the spin orientation of electrons and holes was created by cicularly polarized light. The recombination radiation involving spin-oriented carriers also turns out to be circularly polarized. Therefore the luminescence circular polarization degree $\rho=\left(I^{+}-I^{-}\right) /\left(I^{+}+I^{-}\right)$ can be used to measure the carriers spin polarization and their spin dynamics. Here $I^{+}$and $I^{-}$denote the respective intensities of the left-hand and right-hand circularly polarized luminescence components.

Fig. 2 shows the time dependences $\rho(t)$ measured at four different energies of recombination radiation quanta $E_{\text {det }}$ (marked by arrows in Fig. 1) under excitation into the GaAs barrier $\left(E_{e x c}=1.531 \mathrm{eV}\right)$ with pulse density of $1.6 \mathrm{MW} / \mathrm{cm}^{2}$. It can be seen from Fig. $2, a, b$ that at the high-energy edge of the luminescence line the initial value of $\rho$ is large (it reaches $80 \%$ at $E_{\text {det }}=1.363 \mathrm{eV}$ ) and slowly decreases with a characteristic time of about $300-400 \mathrm{ps}$. When going down to the low-energy part of the luminescence spectrum, the maximum value of $\rho$ significantly decreases and the polarization decay time is drastically reduced (Fig. 2, $c, d$ ). Thus, at $E_{d e t}=1.292 \mathrm{eV}$, $\rho$ decreases from an initial value of $\rho(0) \approx 22 \%$ down to $5 \%$ within 15 ps (Fig. $2, d$ ). 
Let us first make some remarks regarding the nature of the slow decay of $\rho$ at large $E_{d e t}$. The rate of the change of $\rho$ is determined by the slowest of the spin relaxation processes in electrons and holes. Due to the strong spin-orbit interaction in the valence band, the spin relaxation time of holes is significantly shorter than that of electrons $[8]$ and does not exceed a few picoseconds in bulk intrinsic semiconductors of the GaAs type [10]. For this reason we think that the luminescence polarization is only determined by the spin polarization of electrons, while the slow change of $\rho$ is due to the large value of their spin relaxation time.

Quite surprising was the fact that at large $E_{\text {det }}$ during some tens of picoseconds, the values of $\rho$ substantially exceed $50 \%$, reaching $80 \%$ at $E_{\text {det }}=1.363 \mathrm{eV}$ (Fig. 2,a). Since in investigated dots the holes participating in the radiative recombination are heavy holes [11], the value of $\rho$ is numerically equal to the spin polarization of electrons $P_{e}: \rho=P_{e}$ [8]. At the same time, in accordance with the optical selection rules [8], the spin polarization of the electrons generated in bulk GaAs cannot exceed 50\%.

The qualitative explanation of the appearance of $\rho$ values larger than $50 \%$ on the high energy side of the QDs spectra may be obtained in the following simple model. Suppose that the electrons, generated in the GaAs barrier with a spin polarization equal to $50 \%$, are trapped by QDs with such polarization, while the holes lose entirely their polarization during their energy relaxation process. In dots analogous to ours, besides the ground levels there are excited levels of both holes and electrons [6,11-16]. By virtue of Pauli's principle each level can locate no more than two electrons with opposite spins. As at $P_{e}=0.5$ there are thrice as much electrons with spin $-1 / 2$ than electrons with $\operatorname{spin}+1 / 2$, so with the increase of the concentration of photoexcited carriers leading to the saturation of the ground state, the highest electron levels will be populated predominantly by electrons with spin $-1 / 2$, which leads, at the limit, to the $100 \%$ polarization of the associated recombination radiation. The scatter of the QD sizes, resultig in the inhomogeneous broadening of the luminescence line, diminishes this effect; at the high-energy edge of the line, however, the value of $\rho$ may exceed $50 \%$, which is just what we observe in our experiments. On the contrary, the radiation from the ground state must be non-polarized, since two electrons occupying this state have opposite spins. This is also observed experimentally (see Fig. 2, $d$ ).

The long-living and large polarization of electrons can be used for the observation of their spin beats in transverse magnetic field and determination of the g-factor magnitude. Electron spin quantum beats set in under coherent excitation of the two electron spin levels by a short pulse of circularly polarized light and may be considered as a result of Larmor precession of electron spins about the magnetic field $\mathbf{B}$ with a frequency $\boldsymbol{\Omega}=g \mu_{B} \mathbf{B} / \hbar$, where $g$ is the electron $g$-factor, $\mu_{B}$ is the Bohr magneton [17]. The pump pulse is substantially shorter than the electron lifetime and spin relaxation time, denoted $\tau$ and $\tau_{S}$, respectively. Therefore the motion of the average spin $\mathbf{S}$ of electrons excited during the pump pulse

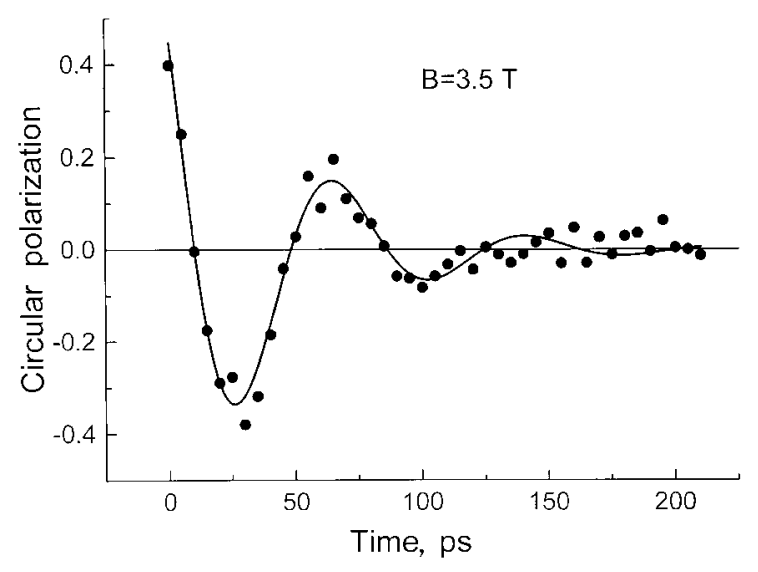

Figure 3. Luminescence polarization oscillations in a transverse magnetic field $B=3.5 T$. $T=1.7 \mathrm{~K}$. The solid line is drawn on formula (2) with Larmor precession period equal to $76 \mathrm{ps}$.

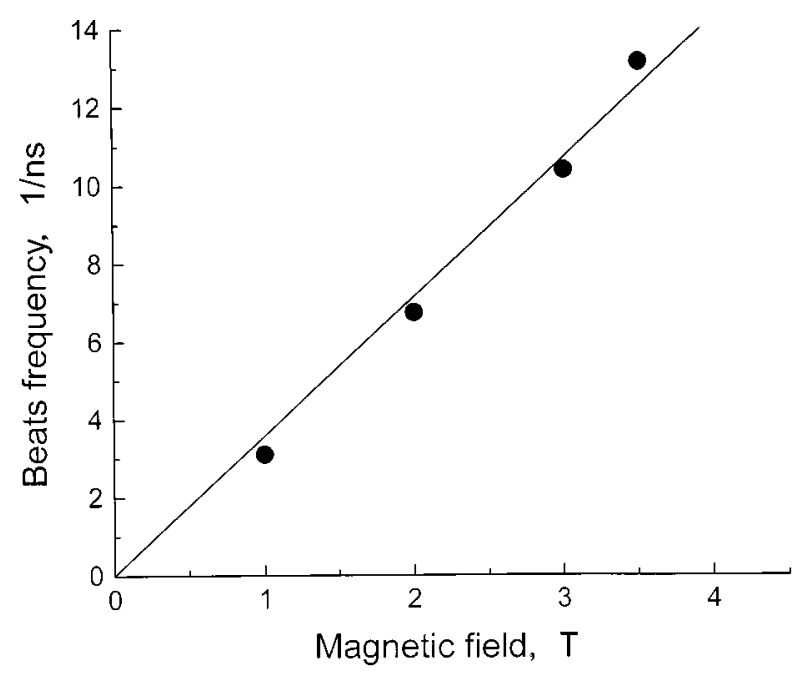

Figure 4. Spin beat frequency versus magnetic field.

obeys the equation

$$
\frac{d \mathbf{S}}{d t}=(\boldsymbol{\Omega} \times \mathbf{S})-\frac{\mathbf{S}}{\tau_{S}},
$$

where the first term on the right-hand side describes the Larmor precession, and the second, spin relaxation. Since in our experiments a long-living luminescence polarization is created by the electron polarization, $\rho=-2 S_{z}$, and in a magnetic field directed perpendicular to the exciting beam, $\rho$ varies in time as

$$
\frac{\rho(t)}{\rho(0)}=e^{-t / \tau_{s}} \cos \Omega t .
$$

It is easy to determine the magnitude of the electron $g$ factor by measuring the beat frequency as a function of the magnetic field.

The dependence $\rho(t)$ measured in a magnetic field $B=3.5 \mathrm{~T}$ at $E_{\text {det }}=1.355 \mathrm{eV}$ is shown in Fig. 3. It 
can be seen that $\rho$ oscillates with time in agreement with Eq. (2). The dependence of the spin beats frequency on the magnetic field is presented on Fig. 4. It is quite linear. Thus the modulus of the electron transverse g-factor of $\mathrm{In}_{0.5} \mathrm{Ga}_{0.5} \mathrm{As} / \mathrm{GaAs}$ QDs can be accurately deduced. We find: $\left|g_{\perp}\right|=0.27 \pm 0.03$. Note that it is the $g$-factor of electrons in the excited state.

To conclude, electron spin orientation and its subsequent dynamics in self-organized $\mathrm{In}(\mathrm{Ga}) \mathrm{As} / \mathrm{GaAs}$ QDs have been studied for the first time using picosecond time-resolved luminescence spectroscopy. It has been found that optical orientation of electrons, excited in the matrix or in the wetting layer, can last after their capture by the QDs during some hundred picoseconds. The saturation of electron ground states at high excitation intensity leads to electron spin polarization in the excited states close to $100 \%$, and to vanishing values in the ground state. Electron spin quantum beats in transverse magnetic field are observed for the first time in semiconductor QDs, leading to determination of the magnitude of the transverse electron $g$-factor in $\mathrm{In}_{0.5} \mathrm{Ga}_{0.5} \mathrm{As} / \mathrm{GaAs}$ QDs.

The authors are grateful to N.N. Ledentzov, V.M. Ustinov, A.E. Zhukov and A.F. Tsatsul'nikov for supplying the samples and useful discussions, I.A. Merkulov and K.V. Kavokin for fruitful discussions.

Partial support of the Russian Fundamental Research Foundation (Grants 96-02-16941 and 98-02-18213) is acknowledged.

\section{References}

[1] G. Wang, S. Fafard, D. Leonard, J.E. Bowers, J.L. Merz, P.M. Petroff. Appl. Phys. Lett. 64, 21, 2815 (1994).

[2] A. Takeuchi, Y. Nakata, S. Muto, Y. Sugiyama, T. Usuki, Y. Nishikawa, N. Yokoyama, O. Wada. Jpn. J. Appl. Phys., Part 2 34, 11a, L1439 (1995).

[3] N. Vollmer, E.J. Mayer, W.W. Ruhle, A. Kurtenbach, K. Eberl. Phys. Rev. B54, 24, R17292 (1996).

[4] R. Heitz, M. Veit, N.N. Ledentsov, A. Hoffman, D. Bimberg, V.M. Ustinov, P.S. Kop'ev, Zh.I. Alferov. Phys. Rev. B56, 16, 10435 (1997).

[5] M. Grundman, D. Bimberg. Phys. Rev. B55, 15, 9740 (1997).

[6] S. Grosse, J.H.H. Sandmann, G. von Plessen, J. Feldmann, H. Lipsanen, M. Sopanen, J. Tulkki, J. Aporelto, Phys. Rev. B55, 7, 4473 (1997).

[7] R. Heitz, A. Kalburge, Q. Xie, M. Grundmann, P. Chen, A. Hoffman, A. Madhukar, D. Bimberg. Phys. Rev. B57, 15, 9050 (1998).

[8] Optical Orientation / Ed. by F. Meier and B. Zakharchenya. In: Modern Problems in Condensed Matter Sciences. NorthHolland, Amsterdam, (1984) v. 8; [Nauka, Leningrad (1989)].

[9] A.Yu. Egorov, A.E. Zhukov, P.S. Kop'ev, N.N. Ledentsov, M.V. Maximov, V.M. Ustinov. Semiconductors, 28, 8, 809 (1994); N.N. Ledentsov, M. Grundmann, N. Kirstaedter, O. Schmidt, R. Heitz, J. Bohrer, D. Bimberg, V.M. Ustinov, V.A. Shchukin, A.Yu. Egorov, A.E. Zhukov, S.V. Zaitsev, P.S. Kop'ev, Zh.I. Alferov, S.S. Ruvimov, P. Werner, U. Gosele, I. Heidenreich. Solid State Electron. 40, 785 (1996).
[10] P. Le Jeune, X. Marie, T. Amand, J. Barrau, R. Planel. Proc. 24th Int. Conf. Phys. Semic. Jerusalem, Israel (1998). To be published.

[11] M. Grundman, O. Stier, D. Bimberg. Phys. Rev. B52, 16, 11969 (1995).

[12] C. Guasch, C.M. Sotomayor Torres, N.N. Ledentsov, D. Bimberg, V.M. Ustinov, P.S. Kop'ev. Superlattices and Microstructures 21, 4, 509 (1997).

[13] R.J. Warburton, C. S. Durr, K. Karrai, J.P. Kottaus, G. Medeiros-Ribeiro, P.M. Petroff. Phys. Rev. Lett. 79, 26, 5282 (1997).

[14] E. Itskevich, I.A. Trojan, S.G. Lyapin, D. Mowbray, M.S. Skolnick, M. Hopkinson, L. Eaves, P.C. Main, M. Henini. Proc. 24th Int. Conf. Phys. Semic., Jerusalem, Israel (1998). To be published.

[15] Craig Pryor. Phys. Rev. Lett. 80, 16, 3579 (1998); Craig Pryor. Phys. Rev. B57, 12, 7190 (1998).

[16] O. Stier, M. Grundmann, D. Bimberg. Proc. 24th Int. Conf. Phys. Semic., Jerusalem, Israel (1998). to be published; O. Stier, M. Grundmann, D. Bimberg. Phys. Rev. B, To be published.

[17] A.P. Heberle, W.W. Ruhle, K. Ploog. Phys. Rev. Lett. 72, 24, 3887 (1994); R.M. Hannak, M. Oestreich, A.P. Heberle, W.W. Ruhle, K. Kohler. Solid State Commun. 93, 4, 313 (1995). 